\title{
Effect of different oxygen concentrations developing pulmonary atelectasis of neonates during anesthesia: a randomized controlled trial
}

\author{
Bo Feng ${ }^{1}$, Li-Ming Cheng ${ }^{1, \star}$, Ming Zhang ${ }^{1}$, Yan Zeng ${ }^{1}$
}

${ }^{1}$ Department of Anesthesiology, Kunming Children's Hospital, 650034 Yunnan, China

\section{*Correspondence}

Medcheng@126.com

(Li-Ming Cheng)

\begin{abstract}
Objectives: This study sought to evaluate the effect of different oxygen concentrations on the development of pulmonary atelectasis in neonates during anesthesia with mechanical ventilation using ultrasound.

Methods: Sixty-three neonates undergoing laparotomies were randomly divided into three groups. A: $\mathrm{FiO}_{2}=30 \%, \mathrm{~B}: \mathrm{FiO}_{2}=40 \%, \mathrm{C}: \mathrm{FiO}_{2}=50 \%$. Ultrasound was performed to guide pulmonary resuscitation maneuvers after tracheal intubation and before tracheal extubation and performed at the end of surgery in each group to evaluate lung consolidation. The following parameters were assessed: (1) primary observation index: pulmonary consolidation score; (2) secondary observation index: area of pulmonary atelectasis, changes in oxygenation index, pulmonary atelectasis, and the incidence of hypoxemia; (3) pulmonary complications at 72 hours postoperatively.

Results: There was a significant difference in pulmonary consolidation scores between the $30 \%$ and $50 \%$ groups $(p<0.001)$ and the $40 \%$ and $50 \%$ groups $(p<0.01)$; the difference between the $30 \%$ and $40 \%$ groups was not significant $(p>0.05)$. The incidence of pulmonary atelectasis was $82.54 \%$. Pulmonary atelectasis occurred in the posterior $(81 \%)$ and in the right posteriolateral positions $(51 \%)$. There was a significant change in the oxygenation index before and after pulmonary ultrasound-guided reexpansion before recruitment (T1) compared to after preoperative recruitment (T2) ( $p$ $<0.001)$ and before postoperative recruitment (T3) compared to after postoperative recruitment (T4) $(p<0.001) .6$ children in the $30 \%$ oxygen concentration group developed mild hypoxemia and 2 children developed moderate hypoxemia; while no hypoxemia occurred in the remaining two groups. No pulmonary complications occurred at 72 hours postoperatively.

Conclusions: $40 \% \mathrm{FiO}_{2}$ may be the oxygen concentration that neither causes significant pulmonary atelectasis nor hypoxemia during the maintenance of mechanical ventilation during neonatal anesthesia. However, there may also be lower lung consolidation scores and no hypoxemia between $30 \%$ oxygen concentration and $40 \%$ we have not studied it. Pulmonary atelectasis is present in most anesthetized patients and occurs mainly in gravity-dependent areas, more on the right than on the left side. Pulmonary ultrasoundguided pulmonary recruitment maneuvers can significantly improve neonatal pulmonary gas exchange function.
\end{abstract}

\section{Keywords}

Lung ultrasound; Pulmonary atelectasis; Neonates; Oxygen; Anesthesia

\section{Introduction}

Pulmonary atelectasis, also known as pulmonary insufflation, is a decrease in the volume and air content of one lung or one or more of its lobes, segments and subsegments, and a collapse of lung tissue. Pulmonary atelectasis leads to decreased lung compliance, impaired oxygenation, increased pulmonary vascular resistance, and lung injury [1]. Pulmonary atelectasis occurs in approximately $90 \%$ of anesthetized patients and can occur under both spontaneous and controlled breathing, whether intravenous or inhalation anesthesia is used [2].

Percent partial pressure of inhaled oxygen is an important factor that is closely related to the level of partial pressure of blood oxygen in neonates during anesthesia. Variations in the level of the percent partial pressure of inhaled oxygen regulate the level of oxygenation in the neonates and, ensure that neonatal perioperative blood oxygen is maintained in the 
normal range. Perioperative oxygen also helps to reduce the incidence of surgical site infections and postoperative nausea and vomiting, but high oxygen concentrations may adversly affect the brain and lungs in neonates [3], and can lead to pulmonary atelectasis [4]. It is estimated that more than $80 \%$ of patients undergoing anesthesia experience "potentially preventable hyperoxemia". The expert consensus on lungprotective ventilation strategies for surgical patients recommends avoiding high concentrations of oxygen and, after that $\mathrm{FiO}_{2}$ (Fractions of Inspired Oxygen Concentration) should be $\leq 0.4$ to maintain normal blood oxygen levels, and that all patients should be given at least $5 \mathrm{~cm} \mathrm{H}_{2} \mathrm{O}$ PEEP [5]. Prolonged exposure to $\mathrm{FiO}_{2}$ of 0.5 or lower is acceptable, while $\mathrm{FiO}_{2}$ above 0.5 will lead to lung injury over time [6].

Ultrasound is widely used in the diagnosis of pulmonary disease. Neonatal pulmonary atelectasis used to be diagnosed based on the child's medical history, clinical presentation and chest X-ray and CT manifestations, but now it can be easily diagnosed with lung ultrasound [7]. Ultrasound has many advantages over X-ray and CT scans, including simplicity, noninvasiveness, avoidance of radiation damage, and low cost. Ultrasound not only confirms the diagnosis of pulmonary atelectasis, but also provides real-time dynamic guidance for pulmonary resuscitation guidance [8], significantly reduces the recurrence rate of pulmonary atelectasis [9].

The number and area of alveoli are much lower in neonates than in adults. The metabolic rate is higher in neonates than in adults based on body weight [10]. Pulmonary insufficiency is likely to be seen when the metabolic needs are increased during surgery. Low oxygen concentrations predispose to hypoxia, while high oxygen concentrations significantly increase the incidence of pulmonary atelectasis. We used ultrasound to assess the relationship between different oxygen concentrations with neonatal pulmonary atelectasis during anesthetic mechanical ventilation and to investigate the effect of pulmonary ultrasound-guided pulmonary recruitment maneuvers on pulmonary gas exchange in neonates.

\section{Materials and methods}

This randomized controlled, patient and sonographer-blinded trial was performed at Kunming Children's Hospital of China, between April 2020 to December 2020. The study population was 63 neonates undergoing laparotomies for digestive tract malformations. This study was approved by the ethical review committee of the Kunming Children's Hospital (No 2020-03197-K01). The research is in accordance with the Helsinki Declaration of 1975, as revised in 2010 Data collection was in accordance with Bavarian law (BayKrG, Art. 27).

Inclusion

(1) Age $\leq 28$ days; (2) full-term neonates with welldeveloped lungs; (3) undergoing surgury with general anesthesia.

Exclusion

(1) History of allergy to halogenated anesthetics; (2) preterm infants with underdeveloped fetal lungs; (3) history of significant cardiovascular, respiratory, central nervous, liver and kidney disease.

\subsection{Randomization}

Sixty-three neonates were randomly divided into three groups $\mathrm{A}, \mathrm{B}$ and $\mathrm{C}$, with 21 neonates in each group, using a randomization software. The oxygen concentration was $\mathrm{A}: \mathrm{FiO}_{2}=30 \%$, B: $\mathrm{FiO}_{2}=40 \%, \mathrm{C}: \mathrm{FiO}_{2}=50 \%$; a PEEP $=5 \mathrm{~cm} \mathrm{H} \mathrm{H}_{2} \mathrm{O}$.

\subsection{Anesthesia and ventilation management}

Standard monitoring during anesthesia include ECG, pulse oximetry, non-invasive blood pressure, body temperature and end-expiratory carbon dioxide. A standardized general anesthesia protocol was used in all patients with atropine $0.01 \mathrm{mg} / \mathrm{kg}$, propofol $3 \mathrm{mg} / \mathrm{kg}$, sufentanil $0.3 \mathrm{ug} / \mathrm{kg}$, and cis-atracurium $0.1 \mathrm{mg} / \mathrm{kg}$. Because of low oxygen reserve in neonates, the oxygen concentration upheld at $100 \%$ during induction of anesthesia. After the jaw relaxed, tracheal intubation was performed, and the fiberoptic bronchoscopy was used to determine the correct position of the tracheal tube. Mechanical ventilation was performed with a tidal volume of $8-10 \mathrm{~mL} / \mathrm{kg}$, respiratory rate adjusted to keep $\mathrm{PETCO}_{2}$ at 35-45 $\mathrm{mmHg}$, oxygen concentration adjusted to the target oxygen concentration. PEEP was given to all neonates at $5 \mathrm{~cm}$ $\mathrm{H}_{2} \mathrm{O}$. Remifentanil $0.2-0.5 \mathrm{ug} / \mathrm{kg} / \mathrm{min}$ and sevoflurane $1 \mathrm{MAC}$ was used to maintain the depth of anesthesia. All patients had a radial artery catheter to monitor invasive arterial blood pressure and collect arterial blood gas.

\subsection{Ultrasound examination}

The lungs were divided into three regions: anterior, lateral and posterior, using the anterior and posterior axillary lines as boundaries. The ultrasound transducer was placed perpendicular or parallel to the rib cage, and each region of the lung was scanned bilaterally. The ultrasound probe was first placed perpendicular to the rib cage to look for pulmonary atelectasis, and then the ultrasound probe was placed parallel to the rib cage to take images at the most obvious point of pulmonary atelectasis. The scoring criteria were: Pulmonary consolidation score $(0$ no consolidation; 1 -minimal parietal consolidation; 2 - small consolidation; 3 -large consolidation). Pulmonary atelectasis was defined as a pulmonary consolidation score $\geq 2$ in any region (Fig. 1).

Because of the low oxygen reserve and high incidence of difficult airways in neonates, there is uncertainty as to the timing of preoxygenation and anesthesia induction, which interferes with preoperative ultrasound findings. Therefore, after tracheal intubation, all neonates underwent pulmonary recruitment maneuvers and ultrasonography was used to ensure that all lung tissue was in a reexpansion state prior to surgery. Different oxygen concentrations were kept in each group during mechanical ventilation, and the occurrence of pulmonary atelectasis during mechanical ventilation using different oxygen concentrations was assessed by ultrasound at the end of the procedure. 


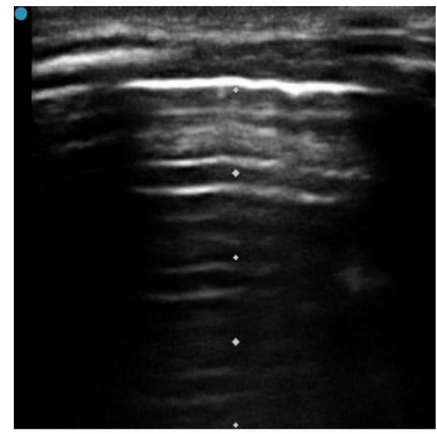

A

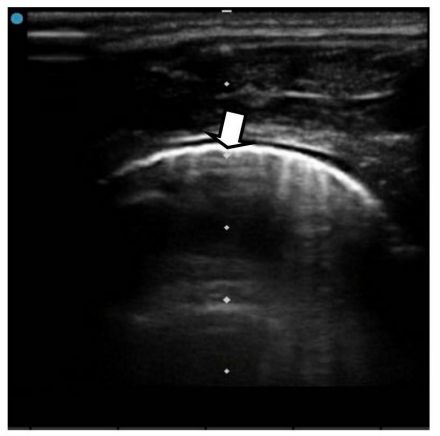

B

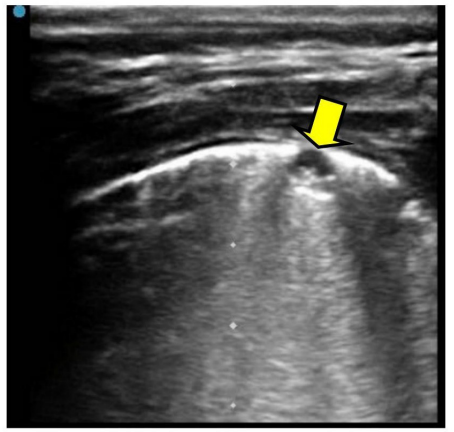

C

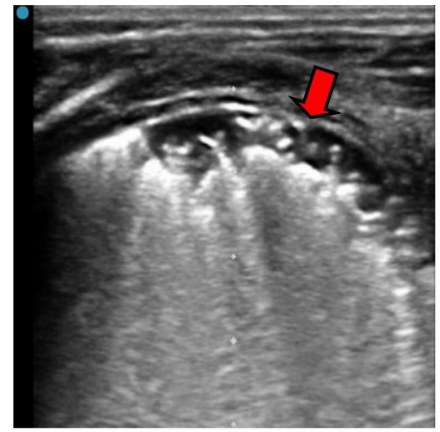

D

F I G URE 1. Reference diagram of lung consolidation score. (A) 0, no consolidation; (B) 1, white arrows indicate minimal pleura consolidations; (C) 2, yellow arrows indicate small-sized consolidations; (D) 3, red arrows indicate large-sized consolidations.

\subsection{Ultrasound-guided recruitment maneuvers (RM)}

Pulmonary recruitment maneuvers (RM) were performed after $10 \mathrm{~min}$ of mechanical ventilation with tracheal intubation, to ensure that there was no preoperative lung tissue collapse. Pulmonary recruitment maneuvers was again performed before extubation of the neonatal to eliminate pulmonary atelectasis due to different oxygen concentrations during mechanical ventilation, to avoid the postoperative effects of identified occurrence of pulmonary atelectasis on the neonates. Ultrasonography was again used to ensure that there was no postoperative lung tissue collapse. During these maneuvers, the oxygen concentration was kept constant in each group and the alveoli were filled with three different oxygen concentrations. Continuous positive airway pressure ventilation was applied, starting at $10 \mathrm{~cm} \mathrm{H}_{2} \mathrm{O}$ and gradually increasing the pressure by $5 \mathrm{~cm} \mathrm{H}_{2} \mathrm{O}$, with each level of continuous positive airway pressure applied for 5 seconds and airway pressure limited to a maximum of $30 \mathrm{~cm} \mathrm{H}_{2} \mathrm{O}$ until there was no collapsed lung area under ultrasound visualization.

\subsection{Blood gas analysis}

To investigate the effect of pulmonary ultrasound-guided pulmonary recruitments strategy on pulmonary gas-exchange function in neonates, arterial blood gases were collected at four time points before and after two pulmonary resuscitations, T1 (before preoperative RM), T2 (after preoperative RM), $\mathrm{T} 3$ (before postoperative RM), and T4 (after postoperative $\mathrm{RM}$ ), respectively, to compare the changes in the oxygenation index.

\subsection{Observed indexes}

Primary observation index: pulmonary consolidation score; secondary observation index: area of pulmonary atelectasis, changes in oxygenation index, pulmonary atelectasis, incidence of hypoxemia, and pulmonary complications (fever, hypoxemia, hypoglossia, laryngospasm, pneumothorax) at 72 hours postoperatively.

\subsection{Double-blind design}

Ultrasound was performed by an experienced ultrasonographer who was blinded to the individual patient groups. Pulmonary areas were scored separately by two blinded investigators. In the event of a scoring disagreement, a third investigator scored the disagreement lung zone lung from the consolidation images. The scorers were only involved in the scoring phase and were not involved in the anesthesia procedures.

\subsection{Statistical analysis}

The study analyzed data using SPSS 21.0 statistical software. The data was expressed as mean \pm standard deviation, or number (percentage) unless otherwise specified. Data was compared within groups by paired samples $t$-test and between groups by the chi-square test; a $p<0.05$ difference was statistically significant.

\section{Results}

From April 2020 to December 2020, 63 newborns were randomized into groups $\mathrm{A}, \mathrm{B}$, and $\mathrm{C}$. Group $\mathrm{A}\left(\mathrm{FiO}_{2}=30 \%\right)$, group $\mathrm{B}\left(\mathrm{FiO}_{2}=40 \%\right)$, and group $\mathrm{C}\left(\mathrm{FiO}_{2}=50 \%\right)$. During the procedure, all patients were in the supine position. The baseline characteristics of patients included in the study are shown in Table 1.

There was a significant difference in pulmonary consolidation scores between the $30 \%$ and $50 \%$ groups ( $p<0.001$ ) and the $40 \%$ and $50 \%$ groups $(p<0.01)$. No statistically significant differences between the $30 \%$ and $40 \%$ groups $(p>$ 0.05 ) (Fig. 2). Pulmonary atelectasis occurred predominantly in the posterior $(81 \%)$ and on the right posterior segments (51\%).

Changes in the oxygenation index were significant before and after ultrasound-guided pulmonary resuscitation at T1 compared to T2 $(p<0.001)$ and at T3 compared to T4 $(p<$ $0.001)$; the decrease in oxygenation index was significant at T2 compared to T3 ( $p<0.001)$ (Fig. 3).

Mild hypoxemia occurred in 6 neonates and moderate hypoxemia in 2 neonates in group A. No hypoxemia occurred in the remaining two groups. In a total of 63 neonates, perioperative pulmonary atelectasis occurred in 52 cases, with an 
TA B L E 1. Baseline characteristics of patients included in the study.

\begin{tabular}{lccc} 
& \multicolumn{3}{c}{ Group } \\
& $30 \%$ group $(\mathrm{n}=13)$ & $40 \%$ group $(\mathrm{n}=13)$ & $50 \%$ group $(\mathrm{n}=13)$ \\
Boys & $10(47.62 \%)$ & $8(38.10 \%)$ & $11(52.38 \%)$ \\
Age, days & $16.62 \pm 11.4$ & $15.62 \pm 8.49$ & $12.46 \pm 7.50$ \\
Weight, $\mathrm{kg}$ & $3.10 \pm 0.57$ & $3.24 \pm 0.42$ & $3.10 \pm 0.41$ \\
\hline Duration of anesthesia, min & $97.53 \pm 12.32$ & $100.48 \pm 11.45$ & $94.72 \pm 13.22$
\end{tabular}

Data are presented as mean \pm standard deviation, or number (\%) as appropriate.

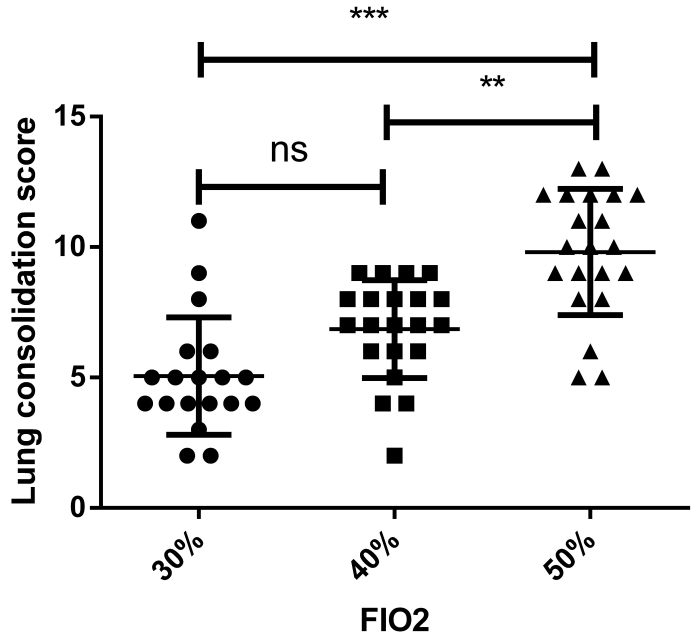

F I GURE 2. The lung consolidation scores (mean \pm standard deviation) under the three groups of different oxygen concentrations, $* * *=p<0.001, * *=p<0.01$, ns $=$ not significant $(p>0.05)$.

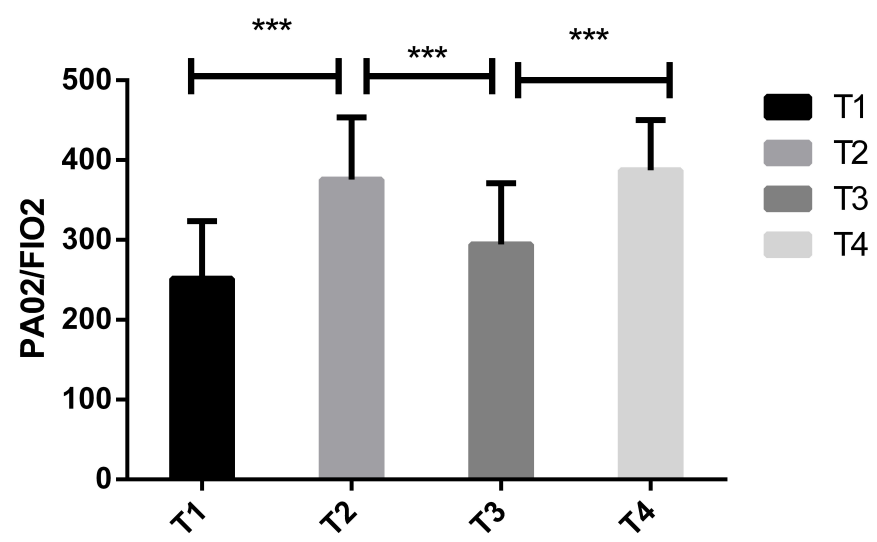

F I G U R E 3. Oxygenation index changes at different time points, $* * *=p<0.001$.

incidence of $82.54 \%$. No pulmonary complications such as fever, hypoxemia, laryngospasm, bronchospasm, pneumothorax were found 72 hours after surgery in the three groups.

\section{Discussion}

In this study, we used ultrasound to assess the relationship between different oxygen concentrations with neonatal pulmonary atelectasis during anesthesia mechanical ventilation.
A two-by-two comparison of lung consolidation scores in groups $\mathrm{A}, \mathrm{B}$, and $\mathrm{C}$ showed statistically significant differences between the oxygen concentration of the $30 \%$ and $40 \%$ groups and the $50 \%$ group, and no statistically significant differences between the $30 \%$ and $40 \%$ groups. The oxygen concentration of the $50 \%$ group had a significantly higher lung consolidation score than the $30 \%$ and $40 \%$ groups. The occurrence of neonatal pulmonary atelectasis is closely related to the concentration of oxygen. As the ventilation/blood flow ratio of the lung is dysregulated, and as the partial pressure of oxygen increases with increasing oxygen concentration, oxygen flows from the alveoli to the capillaries at a faster rate, leading to alveolar collapse [11]. When pulmonary recruitment maneuvers is performed with air, the onset of pulmonary atelectasis is slow and only localized after 40 minutes. However, when the recruitment maneuvers was performed with $100 \%$ oxygen, pulmonary atelectasis reappeared immediately (within 5 minutes) and as long as it was concentrated in the area of pure oxygen resuscitation [12]. With lower oxygen concentrations during maintenance of mechanical ventilation, pulmonary atelectasis still reappeared, but with lower oxygen concentrations compared to higher oxygen concentrations with lower lung consolidation scores. A gradual reduction in oxygen concentration from $100 \%$ to $80 \%$ or $60 \%$ during preoxygenation and subsequent induction of anesthesia reduces the development of pulmonary atelectasis. Over time, the differences in pulmonary atelectasis formation between subjects with different oxygen concentrations became increasingly significant, developing rapidly in patients given $100 \%$ oxygen and slowly in those given lower concentrations [13].

Pulmonary atelectasis occurs predominantly in the posterior segments $(81 \%)$ and most commonly in the right posterior segments $(51 \%)$ during surgery in the horizontal position. In supine mechanically ventilated patients, ventilation is maintained in the abdominal region and cross-lung pressures fall along the gravitational axis, causing the lung to collapse in its dorsalmost aspect [14]. Because the right lung is larger than the left lung in normal patients, with two lobes of the left lung and three lobes of the right lung, the right lung accounts for 55\% and the left lung for $45 \%$ of posterior lung atelectasis, and the right side is more likely to develop atelectasi relative to the left lung. The effect of gravity on respiratory physiology, or more precisely the effect of gravity on trans-pulmonary pressure, has been used to perform a postural pulmonary recruitment maneuvers. The first step is to hypothesize that collapsed lung tissue in the dependent lung region is recruited by placing the patient in the opposite position (i.e., from supine to prone or 
from left to right in the lateral position). The second part uses the stabilizing effect of positive end-expiratory pressure (PEEP) on the airway and alveoli to maintain the open state of the pulmonary region by appropriate PEEP even if the body position returns again to the previous gravity-dependent position of the lung. In this way pulmonary recruitment can be achieved by postural changes without the need to add additional airway pressure as in conventional recruitment maneuvers [15].

We found that pulmonary atelectasis occurred in 52 neonates diagnosed during postoperative ultrasound, with an incidence of $82.54 \%$. This is lower than reported in the literature [16]. which is considered to be related to the preoperative performance of ultrasound-guided pulmonary recruitment maneuvers to eliminate pulmonary atelectasis caused during preoxygenation and induction of anesthesia. The most important purpose of preoxygenation is to increase oxygen reserve in the lungs to increase pulse oximetry tolerance time and delaye onset of hypoxemia during difficult tracheal intubation or ventilation [17], increasing the safety of anesthesia. However, the use of $100 \%$ oxygen without sufficiently aggressive end-expiratory pressure can promote the development of pulmonary atelectasis and shunt during anesthesia [18]. Performing ultrasound-guided pulmonary recruitment maneuvers after tracheal intubation eliminated atelectasis during preoxygenation and induction of anesthesia. Lung recruitment maneuvers were used again before tracheal extubation to eliminate pulmonary atelectasis due to different oxygen concentrations during mechanical ventilation and to avoid the postoperative effects of pulmonary atelectasis in neonates. Within 72 hours after surgery, all three groups of children were free of pulmonary complications such as fever, laryngospasm, bronchospasm, and pneumothorax.

Lowering the oxygen concentrations to prolong the time of appearance of the pulmonary atelectasis reduced the area of pulmonary atelectasis. The nitrogen content of the gas is relatively higher at low oxygen concentrations, and nitrogen is an inert gas not easily absorbed into the blood. A certain volume of nitrogen can prevent collapse of the alveoli, thus reducing the formation of pulmonary atelectasis [19]. However, the risk of hypoxemia increases with a low oxygen concentration. The $30 \%$ oxygen concentration group had 6 cases of mild hypoxemia in neonates, which resolved spontaneously after a few minutes. Two children had moderate hypoxemia, which resolved after pulmonary recruitment. The rest had no pulmonary complications. Hypoxia may cause tissue damage, especially brain tissue damage, and also cardiovascular damage, which can lead to arrhythmias, shock, and even cardiac arrest. The side effects of hypoxia are usually more severe than those of hyperoxia. Therefore, when the risk of hypoxia can be reduced, the inspiratory oxygen concentration should be increased, and a too lower oxygen concentration should not be used simply to reduce the occurrence of pulmonary atelectasis. However, the oxygen concentration should not be increased excessively to avoid the adverse effects of high oxygen concentrations on multiple organs.

The diagnosis of pulmonary atelectasis can be made by chest X-ray and CT scan. The disadvantages of chest Xray and $\mathrm{CT}$ scan are radiation damage and the difficulty of performing bedside examinations in the operating room. Ul- trasound has unique advantages in the diagnosis of pulmonary atelectasis, and has become an important tool for the diagnosis of pulmonary atelectasis because of its convenience, noninvasiveness, high accuracy, and lack of radiation damage. It is also possible to visualize, in real time and in a dynamic state [20]. The resuscitation maneuver reverses the atelectasis and significantly improves pulmonary gas exchange. Significant changes in oxygenation index occurred in T1 compared to T2 and in T3 compared to T4. Pulmonary recruitment maneuvers should be performed intermittently during mechanical ventilation, and the decrease in oxygenation index was significant when comparing T2 and T3. With prolonged ventilation, even with lower concentrations of oxygen, new pulmonary atelectasis reappears, affecting the patient's oxygenation.

In conclusion, pulmonary atelectasis will occur in the vast majority of anesthetized patients, and $40 \%$ oxygen concentration may be the oxygen concentration that neither causes significant pulmonary atelectasis nor hypoxemia during maintenance of mechanical ventilation in neonatal anesthesia. However, there may also be lower lung consolidation scores and no hypoxemia between $30 \%$ oxygen concentration and $40 \%$ we have not studied it. Ultrasound-guided pulmonary recruitment maneuvers facilitate the reopening of collapsed alveoli and significantly improve patient oxygenation. The duration of ventilation increases, even with lower concentrations of oxygen, new pulmonary atelectasis can reappear and affect the patient's oxygenation, so pulmonary recruitment maneuvers should be performed intermittently. Pulmonary atelectasis is most likely to occur in gravity-dependent areas and is more likely to occur on the right posterior. Therefore, after supine surgery, the child can be placed in the lateral position with the ultrasound probe placed in the right dorsal lung region, and the lung recruitment strategy can be implemented under ultrasound guidance in the area most likely to show pulmonary atelectasis until the visualized state of atelectasis is achieved, thus potentially achieving the least pressure and the least number of times to achieve the best results of pulmonary recruitment.

\section{Limitations}

The optimal number of effective perioperative pulmonary recruitments remains to be investigated. Since only three sets of oxygen concentrations were selected, the optimal perioperative oxygen concentration in neonates, i.e., the oxygen concentration with a low incidence of pulmonary atelectasis without perioperative hypoxemia, still needs to be further explored. Postural pulmonary recruitments maneuvers do not require the achievement of high airway pressures and theoretically induce only a mild hemodynamic response that can resolve anesthesiainduced pulmonary atelectasis, and pulmonary gas exchange. Compliance was improved only in the group using the postural change strategy, however, relevant applications in children have been rarely reported. We plan to further explore these issues in future studies.

\section{Conclusions}

Our study has shown that $40 \% \mathrm{FiO}_{2}$ may be the oxygen concentration that neither causes significant pulmonary atelectasis 
nor hypoxemia during maintenance of mechanical ventilation in neonates under general anesthesia. However, there may also be lower lung consolidation scores and no hypoxemia between $30 \%$ oxygen concentration and $40 \%$ we have not studied it. Pulmonary atelectasis is present in most anesthetized patients and occurs mainly in gravity-dependent areas, more on the right than on the left side. Pulmonary ultrasound-guided pulmonary recruitment strategies can significantly improve neonatal pulmonary gas exchange function.

\section{AUTHOR CONTRIBUTIONS}

BF: Data collection and arrangement, Writing-Original draft preparation, Writing-Reviewing and Editing; LMC: Conceptualization, Methodology, Supervision; MZ and YZ: Software, Validation.

\section{ETHICS APPROVAL AND CONSENT TO PARTICIPATE}

This study was approved by the ethical review committee of the Kunming Children's Hospital (No 2020-03-197-K01). The research is in accordance with the Helsinki Declaration of 1975, as revised in 2010 Data collection was in accordance with Bavarian law (BayKrG, Art. 27).

\section{ACKNOWLEDGMENT}

We thank the staff of the Department of Anesthesiology, Kunming Children's Hospital, for their contribution to this study.

\section{FUNDING}

This research received no external funding.

\section{CONFLICT OF INTEREST}

The authors declare no conflict of interest.

\section{DATA AVAILABILITY}

The data used to support the findings of this study are available from the corresponding author upon request.

At the time of data acquisition, the authors were employed at Kunming Children's Hospital, No. 288, Qianxing Road, Xishan District, Kunming City, Yunnan Province, Kunming, Yunnan, China, where the study was conducted.

\section{REFERENCES}

[1] Nestler C, Simon P, Petroff D, Hammermüller S, Kamrath D, Wolf S, et al. Individualized positive end-expiratory pressure in obese patients during general anaesthesia: a randomized controlled clinical trial using electrical impedance tomography. British Journal of Anaesthesia. 2017; 119: 1194-1205.

[2] Tokics L, Strandberg A, Brismar B, Lundqwist H, Hedenstierna G. Computerized tomography of the chest and gas exchange measurements during ketamine anaesthesia. Acta Anaesthesiologica Scandinavica. 1987; 31: 684-692.
[3] Kabon B, Kurz A. Optimal perioperative oxygen administration. Current Opinion in Anaesthesiology. 2006; 19: 11-18.

[4] Lutterbey G, Wattjes MP, Doerr D, Fischer NJ, Gieseke J Jr, Schild $\mathrm{HH}$. Atelectasis in children undergoing either propofol infusion or positive pressure ventilation anesthesia for magnetic resonance imaging. Paediatric anaesthesia. 2007; 17: 121-125.

[5] Young CC, Harris EM, Vacchiano C, Bodnar S, Bukowy B, Elliott $\mathrm{RRD}$, et al. Lung-pro atient: international expert panel-based consensus recommendations. British Journal of Anaesthesia. 2019; 123: 898-913.

[6] Reid MP, Fock A, Doolette DJ. Decompressing recompression chamber attendants during Australian submarine rescue operations. Diving and Hyperbaric Medicine. 2017; 47: 168-172.

[7] Sharma D, Farahbakhsh N. Role of chest ultrasound in neonatal lung disease: a review of current evidences. Journal of Maternal-Fetal \& Neonatal Medicine. 2019; 32: 310-316.

[8] Acosta CM, Sara T, Carpinella M, Volpicelli G, Ricci L, Poliotto S, et al. Lung recruitment prevents collapse during laparoscopy in children: a randomised controlled trial. European Journal of Anaesthesiology. 2018; 35: 573-580.

[9] Yang Y, Geng Y, Zhang D, Wan Y, Wang R. Effect of lung recruitment maneuvers on reduction of atelectasis determined by lung ultrasound in patients more than 60 years old undergoing laparoscopic surgery for colorectal carcinoma: a prospective study at a single center. Medical Science Monitor. 2021; 27: e926748.

[10] H MJ, C BR. Newborn respiration. Obstetrics and Gynecology. 1961; 17: 243-247.

[11] Hedenstierna G, Edmark L. Mechanisms of atelectasis in the perioperative period. Best Practice Research Clinical Anaesthesiology. 2010; 24: 157-169.

[12] Rothen HU, Sporre B, Engberg G, Wegenius G, Högman M, Hedenstierna G. Influence of gas composition on recurrence of atelectasis after a reexpansion maneuver during general anesthesia. Anesthesiology. 1995; 82: 832-842.

[13] Edmark L, Auner U, Enlund M, Ostberg E, Hedenstierna G. Oxygen concentration and characteristics of progressive atelectasis formation during anaesthesia. Acta Anaesthesiologica Scandinavica. 2011; 55: 7581.

[14] Tusman G, Böhm SH. Prevention and reversal of lung collapse during the intra-operative period. Best Practice Research Clinical Anaesthesiology. 2010; 24: 183-197.

[15] Acosta CM, Volpicelli G, Rudzik N, Venturin N, Gerez S, Ricci L, et al. Feasibility of postural lung recruitment maneuver in children: a randomized, controlled study. Ultrasound Journal. 2020; 12: 34.

[16] Tusman G, Acosta CM, Böhm SH, Waldmann AD, Ferrando C, Marquez MP, et al. Postural lung recruitment assessed by lung ultrasound in mechanically ventilated children. Critical Ultrasound Journal. 2017; 9: 22.

[17] Edmark L, Kostova-Aherdan K, Enlund M, Hedenstierna G. Optimal oxygen concentration during induction of general anesthesia. Anesthesiology. 2003; 98: 28-33.

[18] Coussa M, Proietti S, Schnyder P, Frascarolo P, Suter M, Spahn DR, et al. Prevention of atelectasis formation during the induction of general anesthesia in morbidly obese patients. Anesthesia and Analgesia. 2004; 98: 1491-1495.

[19] Song I, Kim E, Lee J, Kang P, Kim H, Kim J. Utility of perioperative lung ultrasound in pediatric cardiac surgery: a randomized controlled trial. Anesthesiology. 2018; 128: 718-727.

[20] Ullmann N, D'Andrea ML, Gioachin A, Papia B, Testa MBC, Cherchi $\mathrm{C}$, et al. Lung ultrasound: a useful additional tool in clinician's hands to identify pulmonary atelectasis in children with neuromuscular disease. Pediatric Pulmonology. 2020; 55: 1490-1494.

How to cite this article: Bo Feng, Li-Ming Cheng, Ming Zhang, Yan Zeng. Effect of different oxygen concentrations developing pulmonary atelectasis of neonates during anesthesia: a randomized controlled trial. Signa Vitae. 2022;18(1):96-101. doi: $10.22514 /$ sv.2021.093. 